\title{
Bonding interactions between ligand-decorated colloidal particles
}

\author{
Tine Curk* \\ Institute of Physics, Chinese Academy of Sciences, 100190 Beijing, China and \\ Department of Chemistry, University of Maribor, Maribor, Slovenia \\ Urban Bren \\ Department of Chemistry, University of Maribor, Maribor, Slovenia \\ Jure Dobnikar ${ }^{\dagger}$ \\ Institute of Physics, Chinese Academy of Sciences, 100190 Beijing, China and \\ Department of Chemistry, University of Cambridge, Cambridge, UK
}

\begin{abstract}
We study the interactions between particles forming reversible bonds. Theoretically we find that the mean association constant between a pair of such particles can be well approximated by a double exponential function of the individual bond free energy in two cases: i) when the individual bond strength is sufficiently weak and ii) when the bond forming ligands are randomly (Poisson) distributed among the particles in the system. In experiments with ligand-grafted colloidal particles, randomness both in number of the ligands per colloid and in their spatial distribution on the colloid is inherent to common fabrication techniques. We theoretically investigate the effect of quenched disordered ligand positions on the interaction heterogeneity. Furthermore, we perform Monte Carlo simulations of colloidal particles decorated with mobile, quenched-disordered or uniformly distributed ligands to investigate to what extent the mean association constant is a good approximation for the magnitude of the effective interactions in such systems, and to define the limits of applicability of the analytical expression. We find that for large enough particles (100 $\mathrm{nm}-$ $1 \mu \mathrm{m}$ in diameter) at usual experimental parameters, the simple analytical expression provides a good description of the inter-particle interactions. The theory is finally extended beyond dimeric bonds to general multimeric complexes.
\end{abstract}

\section{INTRODUCTION}

Ligand-coated colloids have been studied extensively in experiments for the past 20 years (e.g. DNAcoated colloids [1-5]) and sophisticated complex models describing the binding interactions have been developed [6]. The colloidal interactions generally consist of several terms of various strength and complexity. Most interaction types are non-specific, e.g. electrostatic, dipolar, fluid-mediated, steric etc.. Colloids decorated with ligands that can form bonds also interact via specific bonding interactions due to binding of the ligands on different colloids. In this manuscript, we do not discuss any non-specific interaction terms but rather focus on the description of the specific inter-colloidal bonding interactions. Bonding interactions clearly depend on the spatial distribution and mobility of the ligands grafted on the colloids. A theory of valence-limited interactions has been developed that explicitly takes into account specific positions and different types of tethered ligands [6]. It requires a self-consistent solution of a system of equations, see Refs. [7, 8] for quenched (immobile) and [9] for mobile ligands. A complementary approach is based on a saddle-point approximation for the binding free energy [10].

As we demonstrate here, in certain limits the average bonding interactions in such systems can be well approximated by a simple analytical expression featuring a double exponential dependence on the individual bond free energy. Specifically, the double exponential expression is generally valid either for Poisson distributed ligands or for an arbitrary distribution of the ligands provided that the individual bonds are sufficiently weak. We argue that in usual experimental setups employing ligand-coated particles larger than a few tens of nanometers the inter-colloidal interaction strength is usually weaker than $-30 k_{\mathrm{B}} T$ and typically at least one of the above conditions is satisfied. Therefore, the approximations underlying the analytical expression are often justified. However, in order to decide whether or not the double exponential expression can be used to describe the effective interactions and to predict macroscopic

*Electronic address: tc387@cam.ac.uk

$\dagger$ Electronic address: jd489@cam.ac.uk 


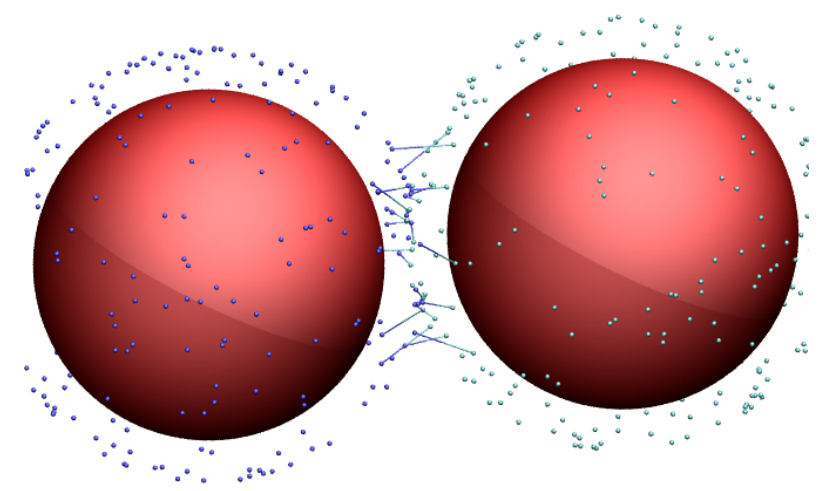

FIG. 1: System snapshot. The two colloids are coloured red, ligand grafting positions are shown as blue and cyan points on the left and right colloid, respectively. A bond formed between two ligands on the opposite colloids is represented with a straight line connecting two ligand grafting points. Note that for visualisation purpose the diameter of red colloids is reduced in the snapshot, the ligand grafting points are positioned on the colloid surface.

properties of such systems, we also need to understand the level of heterogeneity in the system. Namely, if the pair interactions are distributed in a non-homogeneous way, the average interaction strength is not sufficient to describe the physics of the system.

We explore the bonding interactions between a pair of particles using theoretical calculations verified by Monte Carlo simulations. We derive the analytical approximation when individual ligands form dimeric complexes (bonds) and also for multimeric complexes where three or more ligands form a complex $[11,12]$. Finally, we explore the limits of validity of the analytical approach and provide a schematic phase diagram for applicability of the simple analytical pair interaction.

\section{MODEL}

We consider spherical hard-sphere particles of radius $R_{\mathrm{p}}$, each particle having $N$ binders distributed over its surface, see Figure 1. Binders can be e.g. ligands on the surface of the colloidal particles or single stranded DNA segments grafted to it; throughout the text we use both "ligand" or "binder" to describe the same. These binders are attached to the grafting points on the colloid surface with a polymeric spacer. Two binders on different colloids can form a link (bond) whose free energy consists of a term due to the free energy of the bond formation, and a configurational penalty due to stretching of spacers

$$
\Delta G_{i j}=\beta \Delta G_{0, i j}+\Delta G_{\mathrm{cnf}}\left(\mathbf{r}_{i}, \mathbf{r}_{j}\right)
$$

with $\beta \Delta G_{0, i j}$ the hybridisation free energy of the two binders in solution. $\Delta G_{\mathrm{cnf}}\left(\mathbf{r}_{i}, \mathbf{r}_{j}\right)$ is the configurational contribution which only depends on the grafting points $\mathbf{r}_{i}, \mathbf{r}_{j}$ of the two binders, respectively, under the assumption that $\Delta G_{i j}$ does not depend on the presence of other binders. For ideal polymeric spacers, $\Delta G_{\mathrm{cnf}}\left(\mathbf{r}_{i}, \mathbf{r}_{j}\right)$ becomes a quadratic function of the distance $\left|\mathbf{r}_{i}-\mathbf{r}_{j}\right|$. Bonding configurations are valence limited, every binder can attach to at most one other binder. There are no other interactions considered between binders and colloids. Inter-colloidal repulsion is described by a hard sphere potential.

Our aim is to predict the pair interaction free energy between the colloidal particles. We consider three different scenarios of ligand distribution on the colloidal surface: mobile where the grafting points are free to move on the surface of the particle, quenched with random positions on the colloid surface and uniform lattice approximately described by a solution to a Thomson problem [13].

\section{DOUBLE EXPONENTIAL}

Thermodynamic properties of a statistical ensemble do not depend on the value of the diffusion constant as long as it remains nonzero; $D>0$. However, as we show in ref. [14], the ensemble average of the 
colloidal pair interaction remains the same for both immobile $(D=0)$ and mobile $(D>0)$ ligands if the ensemble properties of ligand distribution on isolated particles are the same for both cases. We therefore provide derivation of the mean interaction only for mobile binders, relying on the above argument stating that the result is equally applicable to quenched ligands.

We consider ensemble of particle pairs separated by a distance $d$. The two particles, labelled A and $\mathrm{B}$, are grafted with $n_{\mathrm{A}}$ and $n_{\mathrm{B}}$ binders, respectively. For mobile ligands with uncorrelated positions, the partition function counting all possible linking arrangements at a fixed distance $d$ between the two particles is

$$
Q_{b}\left(n_{\mathrm{A}}, n_{\mathrm{B}}, \epsilon \mid d\right)=\sum_{\lambda=0}^{n_{\mathrm{A}}} \Omega(\lambda) e^{-\beta \epsilon(d) \lambda} .
$$

Here $\Omega(\lambda)$ is the number of distinct linking configurations, $\epsilon(d)$ the effective single bond free energy averaged over all possible grafting point positions, and $\beta \equiv 1 /\left(k_{\mathrm{B}} T\right)$ the inverse temperature. We can write

$$
\Omega(\lambda)=\left(\begin{array}{c}
n_{\mathrm{A}} \\
\lambda
\end{array}\right)\left(\begin{array}{c}
n_{\mathrm{B}} \\
\lambda
\end{array}\right) \lambda !
$$

because we need to choose $\lambda$ bonds out of $n_{\mathrm{A}}$ ligands, $\lambda$ bonds out of $n_{\mathrm{B}}$ ligands and there are $\lambda$ ! ways of binding the chosen ligands together $[15,16]$. The single bond strength

$$
e^{-\beta \epsilon(d)}=\frac{1}{S_{\mathrm{A}} S_{\mathrm{B}}} \int_{S_{\mathrm{A}}} d \mathbf{r}_{1} \int_{S_{\mathrm{B}}} d \mathbf{r}_{2} e^{-\beta \Delta G\left(\mathbf{r}_{1}, \mathbf{r}_{2}\right)},
$$

is integrated over particle surfaces $S_{\mathrm{A}}$ and $S_{\mathrm{B}}$, keeping the inter-particle distance $d$ fixed. Approximate solution for the particle interaction free energy $\beta F_{b}=-\ln \left(Q_{b}\right)$ due to bond formation, as formulated by Angioletti et. al.[6,9] for mobile or immobile ligands, involves self-consistently solving a set of equations.

The binder-coated particles are usually manufactured by some sort of a random grafting process [25]. If the final grafting density is low, such that the inter-binder steric repulsion can be neglected, the resulting number of ligands per particle is expected to be Poisson distributed, i.e. the probability that the particle holds exactly $n$ binders is

$$
p(n)=e^{-\tilde{n}} \frac{\tilde{n}^{n}}{n !},
$$

where $\tilde{n}$ is the average number of binders per particle. The interaction between a specific pair of particles is determined by Eq. (2). But in practice the experiments on ligand-coated colloids are usually performed in a solution containing many particles and thus tend to probe ensemble averages. The ensemble-averaged interaction has a double exponential form as a function of the single bond free energy $\epsilon(d)$ :

$$
\begin{aligned}
\left\langle Q_{b}\right\rangle(\epsilon \mid d) & =\sum_{n_{\mathrm{A}}} \sum_{n_{\mathrm{B}}} p\left(n_{\mathrm{A}}\right) p\left(n_{\mathrm{B}}\right) Q_{b}\left(n_{\mathrm{A}}, n_{\mathrm{B}}, \epsilon \mid d\right) \\
& =\exp \left(\tilde{n}_{\mathrm{A}} \tilde{n}_{\mathrm{B}} e^{-\beta \epsilon(d)}\right) .
\end{aligned}
$$

The detailed derivation is provided in the Online SI where we also show that the formula generalises to different binder types $\left\langle Q_{b}\right\rangle(\boldsymbol{\epsilon} \mid d)=\exp \left(\sum_{i, j} \tilde{n}_{\mathrm{A}, i} \tilde{n}_{\mathrm{B}, j} e^{-\beta \epsilon_{i j}(d)}\right)$, with $\boldsymbol{\epsilon}$ the matrix specifying interactions between all binder types. If the grafting density is large, the excluded volume of individual binders results in a more homogeneous distribution of the ligands on the particles. However, if the aim is to keep the total particle-particle interactions within a certain magnitude range, the individual bonds need to be weaker for larger grafting densities. In the Online SI we show that the same double exponential expression, Eq. (6) applies also in the limit of weak bonds, regardless of the binder number distribution or the spatial correlations of the binder grafting points on the particle's surface.

\section{POTENTIAL OF MEAN FORCE AND PAIR INTERACTION}

The main result of this paper is that for Poisson distributed binders, or weak bonds, the ensemble potential of mean force due to bond formation is

$$
\beta F_{b}(d)=-\ln \left(\left\langle Q_{b}\right\rangle\right)=-\sum_{i, j} \tilde{n}_{\mathrm{A}, i} \tilde{n}_{\mathrm{B}, j} e^{-\beta \epsilon_{i j}(d)},
$$


a sum over all distinct binder types on the particles $\mathrm{A}$ and $\mathrm{B}$, with $\tilde{n}_{\mathrm{A}, i}$ and $\tilde{n}_{\mathrm{B}, j}$, respectively, the mean number of binders of type $i$ and $j$ on particle A and B. Each element of the interaction matrix $\epsilon_{i j}(d)$ is determined by Eq. (4). Note that for large distance $d$ the single bond free energy $\beta \epsilon(d)$ becomes a large positive number and the interaction vanishes $\beta F_{b}(d \rightarrow \infty)=0$. As shown in the Online SI, this formula also generalises to multimeric complexes [11, 12], where three or more binders can interact simultaneously.

Furthermore, as derived in the SI, the potential of mean force, Eq. (7), is equal to the average number of formed bonds

$$
\beta F_{b}(d)=-\langle\lambda\rangle_{d}
$$

$\langle\lambda\rangle_{d}$ is the mean number of distinct bridges connecting particles A and B at distance $d$. Each bridge contributes $1 k_{\mathrm{B}} T$ analogous to the equipartition. This result was found previously [3, 17] for the case of weak interaction where every ligand has a very low probability of being bound. Here we have shown that for ensemble-averaged potential of mean force the same formula extends to Poisson distributed ligands, as well as, multi-meric complexes.

The above relations, Eqs. (6-8), hold for both mobile and immobile ligands. In the case of mobile ligands the interaction will be homogeneous between all colloids if the average number of bonds is a small fraction of the total mean number of ligands $\lambda / n_{\mathrm{A}}, \lambda / n_{\mathrm{B}} \rightarrow 0$. An example would be interaction between patches of big lipid bilayers providing an infinite reservoir of binders to the interacting regions. In this case the heterogeneity of the interaction is negligible and Eqs. (6-8) directly describe the pair interaction. On the other hand, if the fraction of formed bonds is appreciable $\left(\lambda / n_{\mathrm{A}} \sim 1\right)$, interaction heterogeneity is appreciable as well because the polydispersity in the number of binders is quenched. In this case, however, many body effects also become important and the pair interaction is not sufficient to characterise the system [7].

For immobile (quenched disorder) ligands the heterogeneity of interactions can be appreciable even when the fraction of formed bonds approaches zero $\left(\lambda / n_{\mathrm{A}} \rightarrow 0\right)$. For example, in the case of very short linkers the ensemble average of the interaction, Eq. (6), is dominated by a rare interaction between two particles that happen to have many binders grafted on complementary positions. For such cases the ensemble average is still given by Eqs. (6) and (7), but the heterogeneity (variance in the pair interaction) also plays a major role. Very large heterogeneity of interaction is usually not desired in applications because s vast number of particle pairs will exhibit weak interaction, while only a few pairs will dominate. A degree of heterogeneity, however, can be beneficial for colloidal crystal formation because the strong-binding pairs form the nucleus which can then grow by the addition of the weakerbinding particles $[18]$.

For experiments performed on a suspension of particles in solution the relevant quantity is usually the total particle-particle interaction free energy $\beta \Delta F^{p p}$, referenced to the standard molar concentration $\rho_{0}=1 \mathrm{M}$. It can be obtained by integrating Eq. (7),

$$
e^{-\beta \Delta F^{p p}}=\rho_{0} N_{\mathrm{A}} \int_{0}^{\infty} 4 \pi x^{2} e^{-\beta F_{b}(x)} d x,
$$

with $x$ the inter-particle centre-of-mass distance and $N_{\mathrm{A}}$ the Avogadro's number. This integration, in general, must be performed numerically. However, as we show in the SI if the particle is large compared to the thermal linker length (usually the case with DNA-coated colloids), we can employ a mean-field approximation

$$
\beta \Delta F_{\mathrm{mf}}^{p p}=-\sum_{i, j} \tilde{n}_{\mathrm{A}, i} \tilde{n}_{\mathrm{B}, j} e^{-\beta \epsilon_{i j}^{*}}-\beta \Delta F_{0}^{p p},
$$

where the second term is the reference free energy. Choosing $\beta \Delta F_{0}^{p p}=\ln \left(4 \pi\left(2 R_{\mathrm{p}}\right)^{2} \delta x \rho_{0} N_{\mathrm{A}}\right)$ would ensure that $\Delta F^{p p}$ is normalised with respect to the standard concentration $\rho_{0}$, with $\delta x$ the single bond thermal fluctuating distance. For harmonic bonds with stiffness $k$ we obtain $\delta x=(\beta k)^{-0.5}$. The meanfield expression, Eq. (10), assumes that for every link the integration over the inter-particle distance $d$ can be performed independently. This approximation is expected to be valid if the links are very short compared to the particle size $\delta x / R_{\mathrm{p}} \rightarrow 0$ such that the fluctuations in $d$ can be neglected. The effective single bond strength $\epsilon^{*}$ can be calculated by integration

$$
e^{-\beta \epsilon_{i j}^{*}}=\frac{1}{4 R_{\mathrm{p}}^{2} \delta x} \int_{0}^{\infty} x^{2} e^{-\beta \epsilon(x)} d x
$$


or simply fitted from experiments. Note that if $\Delta G_{\mathrm{cnf}}$ is the same for all binder pairs $i j$ we can write, using Eqs. (1) and (4), $\epsilon_{i j}^{*}=\Delta G_{0, i j}+\Delta G_{\mathrm{cnf}}^{*}$, with the integrated configuration contribution $\Delta G_{\mathrm{cnf}}^{*}$ a common constant that can be either calculated by numerical integration, if the functional form of $\Delta G_{\mathrm{cnf}}\left(\mathbf{r}_{i}, \mathbf{r}_{j}\right)$ is known, or fitted from experimental data.

\section{MC SIMULATIONS}

To verify and extend the above theoretical derivations, we perform Monte Carlo simulations of a pair of ligand-decorated particles and calculate the inter-particle interaction free energy due to bond formation. We simulate two hard-sphere particles of radius $R_{\mathrm{p}}$, each having $N$ binders grafted to its surface, as depicted by a simulation snapshot on Figure 1. The binders on a given particle are noninteracting and we consider three cases: (i) mobile binders, (ii) random quenched binders; where the specific grafting location of each binder on the particle is chosen randomly on the surface of the particle, and (iii) uniformly distributed binders; where the position of the binders is obtained by the solution of the Thomson problem [13]. Two binders on different particles can form a bond whose energy consists of a term due to the free energy of the bond formation, $f_{0}$, and a harmonic energy penalty due to stretching of the polymers:

$$
\Delta G\left(\mathbf{r}_{i}, \mathbf{r}_{j}\right)=f_{0}+\frac{k}{2}\left|\mathbf{r}_{i}-\mathbf{r}_{j}\right|^{2}
$$

Here $k$ is the stiffness of the bond and $\mathbf{r}_{i}$ and $\mathbf{r}_{j}$ are the binder grafting points locations. This model maps to the analytical model discussed above with simple mapping $\Delta G_{\mathrm{cnf}}\left(\mathbf{r}_{i}, \mathbf{r}_{j}\right)=\frac{k}{2}\left|\mathbf{r}_{i}-\mathbf{r}_{j}\right|^{2}+\Delta G_{\mathrm{cnf}}\left(\mathbf{r}_{i}=\mathbf{r}_{j}\right)$ and $f_{0}=\Delta G_{0}+\Delta G_{\mathrm{cnf}}\left(\mathbf{r}_{i}=\mathbf{r}_{j}\right)$. Bonding interactions are valence limited, every binder can attach to at most one other binder. Inter-particle repulsion is described by a hard sphere potential and there are no other interactions considered between binders and particles.

We perform Monte Carlo (MC) simulations of a pair of particles, employing standard MC moves such as particle rotation and translation. The bond formation and swapping moves are performed by employing a form of configurational bias Monte Carlo [19] described in ref. [14]. The free energy as a function of the number of formed bonds $\lambda$ is calculated using the Wang-Landau technique [20] by biasing the sampling in the number of formed bonds. The free energy reference is chosen as the two unbound particles within a surface-to-surface distance $\delta x=(\beta k)^{-0.5}$, such that the free energy obtained from simulations can be directly compared with the analytical expression (10) with $\beta \Delta F_{0}^{p p}=0$. The Wang-Landau technique is used to calculate the potential of mean force between a pair of particles by confining both particle on a line and biasing the sampling in the inter-particle distance with bin size $a=\frac{1}{4 \sqrt{\beta k}}$.

Figure 2 shows the potential of mean force obtained from Monte Carlo simulations and analytical theory. In the case of mobile ligands the agreement is very good. The bond strength is chosen to be relatively strong such that the effects of quenched disorder, and resulting heterogeneity of interaction, are clearly visible.

The interaction free energy as a function of the number of formed bonds between the two parties obtained from Monte Carlo simulations and the analytical theory is shown in Figure 3. Only the configurational free energy is shown $F_{\mathrm{cnf}}^{p p}(\lambda)=F^{p p}\left(\lambda \mid f_{0}=0\right)$, however, any value for the total interaction can be obtained by a linear mapping $F^{p p}(\lambda)=F_{\mathrm{cnf}}^{p p}(\lambda)+\lambda f_{0}$ and $F^{p p}=\sum_{\lambda} F^{p p}(\lambda)$.

In the case of mobile ligands, the analytical mean-field model works very well in a wide range of formed links $\lambda$ (blue symbols and solid curves on Figure 3). For a large particle radius $R^{*}=R \sqrt{\beta k}$ the agreement becomes almost exact. In the case of quenched disorder (red stars), however, the interaction shows variation depending on the particular random realisation of binder grafting point locations. The exponential average of the pair interaction is still given by Eq. (9). For large number of bonds $\lambda$, however, most of the realisations will result in a significantly weaker interaction because the exponential average is dominated by a rare configuration. Heterogeneity of the interaction is low only if the number of formed bonds is sufficiently low $\lambda<\lambda^{*}$, where we have denoted with $\lambda^{*}$ the limiting number of bonds for which interactions are sufficiently homogenous (this definition is of course somewhat arbitrary, below we give a possible criterium, which we chose to work with). If the ligands are uniformly distributed, the limit $\lambda^{*}$ is even lower. 


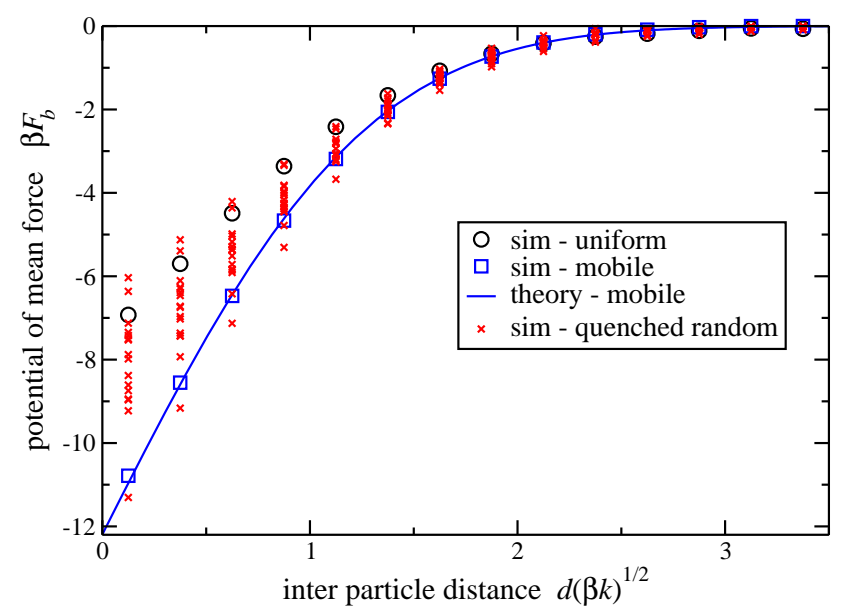

FIG. 2: Potential of mean force between two particles obtained from Monte Carlo simulations (symbols) and analytical theory (solid curve) using Eq. (7). The quenched disorder data (red starts) shows the calculated interactions for 16 different random realisations of grafting points positions. Parameters: particle radius $R \sqrt{\beta k}=50$, bond strength $\beta f_{0}=-5$, number of ligands per particle

$$
N=n_{\mathrm{A}}=n_{\mathrm{B}}=256 \text {. }
$$

\section{HOMOGENEOUS INTERACTION CONDITION}

In the following, we aim to determine the bound on particle physical parameters (such as size, ligand density, total interaction strength) at which the inter-particle interaction is expected to be homogenous for immobile binders with random quenched grafting points locations. Specifically, we determine the limiting number of bonds $\lambda^{*}$ for which we expect to find at least one inter-particle orientation at which the quenched immobile binders would yield a similar interaction strength as mobile binders.

First, we determine the size of the spherical cap within which bound ligands are found in the case of mobile ligands. When multiple bonds $\lambda$ are formed the distance fluctuations of the two particles $d_{c} \ll \delta x$ will be smaller than the distance fluctuations (perpendicular to the particle surface) of individual bonds: $\delta x \approx \sqrt{k_{\mathrm{B}} T / k}$ for harmonic bonds. Therefore, we can neglect the inter-particle distance fluctuations and assume the interacting spherical cap size is determined by the single bond extension $h_{c}=\frac{\delta x}{2}$. The fraction of the total colloid surface covered by the cap is $f_{s}=h_{c} / 2 R_{p}$ with $R_{p}$ the particle radius, yielding a lateral cap radius of $r^{\text {cap }}=2 R_{p} \sqrt{f_{s}\left(1-f_{s}\right)}$.

In addition to bonded ligands being present within the interacting spherical cap, the lateral positioning of the two bonded ligands is correlated. The typical lateral correlation length is of the size of a typical thermal bond distance $\delta x$, for harmonic bonds $\delta x \approx \sqrt{k_{\mathrm{B}} T / k}$. These two conditions: (i) all bonded ligands are confined to a spherical cap and (ii) the positions of bonded ligands are laterally confined, represent typical configurations obtained with mobile ligands. We rationalise that, if such configurations are expected to be present for all pairs of particles with quenched immobile ligands, the pair interaction in the quenched case would be homogenous.

The probability that two specific patches (of size $f_{s}$ ) on the two particles are holding $k_{1}$ and $k_{2}$ ligands, respectively, is a product of the two Poisson distributions $p\left(k_{1}, k_{2} \mid \bar{k}\right)=p\left(k_{1} \mid \bar{k}\right) p\left(k_{2} \mid \bar{k}\right)$, with $p(k \mid \bar{k})$ a Poisson distribution, $\bar{k}=f_{s} N$ the mean number of ligands per patch and $N$ the total mean number of ligands per particle. There are $1 / f_{s}$ such distinct patches on each particle and all possible orientations of the two particles must be considered. Therefore, the expected number of distinct patch pairs holding exactly $k_{1}$ and $k_{2}$ ligands on the first and second particle, respectively, is $\left\langle n^{p p}\right\rangle_{k_{1}, k_{2}}=\frac{1}{f_{s}^{2}} p\left(k_{1}, k_{2} \mid \bar{k}\right)$

The probability that a quenched randomly positioned ligand within the interacting patch will be able to find a complementary ligand randomly positioned on the second particle is $p^{\text {pair }} \approx\left(\delta x / r^{\text {cap }}\right)^{2}$, i.e. the ratio of $\pi(\delta x)^{2}$ to the patch area. The relative positioning of the two colloidal patches has two lateral and one rotational degree of freedom. Therefore, the first bond can always be formed $p^{\text {pair }}(\lambda=1)=1$, 


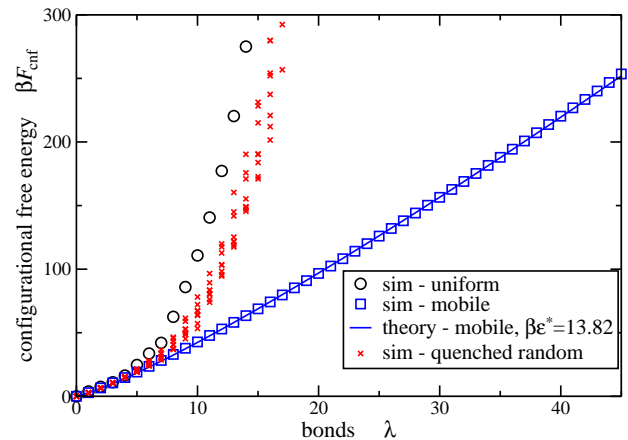

(a) $R^{*}=50, N=256$

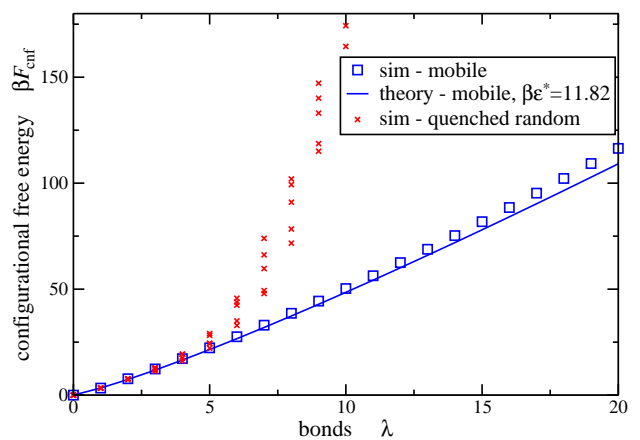

(c) $R^{*}=20, N=48$

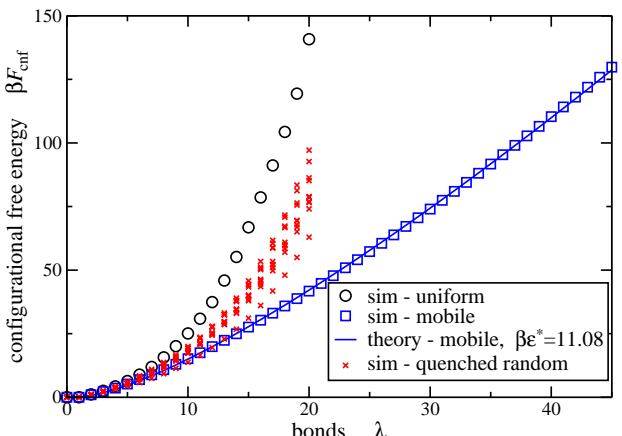

(b) $R^{*}=20, N=256$

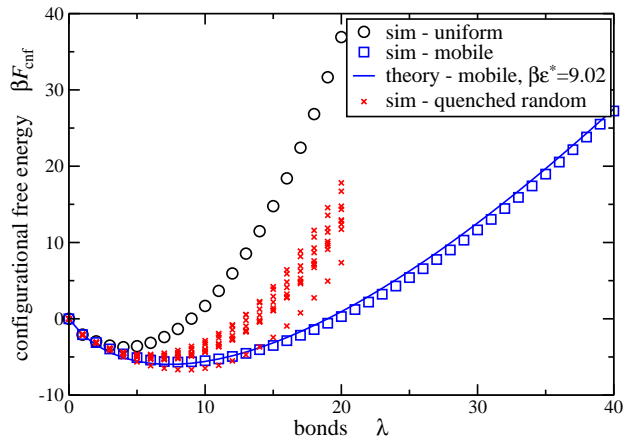

(d) $R^{*}=10, N=256$

FIG. 3: Total configurational free energy $F_{\mathrm{cnf}}^{p p}(\lambda)=F^{p p}\left(\lambda \mid f_{0}=0\right)$ as a function of the number of bonds obtained from Monte Carlo simulations (symbols) and analytical theory (solid curves), using Eq. (10), where the single bond free energy $\epsilon^{*}$ was determined by numerically solving the integral, Eq. (11), at $f_{0}=0$. The reduced particle radius $R^{*}=R /(\delta x)=R \sqrt{\beta k}$ is normalised by the typical bond length. The quenched disorder data (red starts) shows the calculated interactions for multiple different random realisations of grafting points positions. The theoretical curve is obtained by expanding Eq. (10) in a Taylor series over the number of formed bonds: $e^{-\beta F_{\mathrm{mf}}^{p p}(\lambda)}=e^{\beta F_{0}^{p p}}\left(\tilde{n}_{\mathrm{A}} \tilde{n}_{\mathrm{B}} e^{-\beta \epsilon^{*}}\right)^{\lambda} / \lambda$ !.

while the second bond must allow for the rotational freedom of the two patches $p^{\text {pair }}(\lambda=2) \approx \delta x / r^{\text {cap }}$, i.e. the probability that a ligand falls within a circular band with radius $r^{\text {cap }} / 2$ and width $2 \delta x$. In total, the probability that $\lambda$ ligands are positioned near (i.e. within $\delta x$ lateral distance of) ligands on the complementary patch is

$$
p^{\text {near }}(\lambda)=\prod_{i=1}^{\lambda} p^{\text {pair }}(i)=\left(\frac{\delta x}{r^{\text {cap }}}\right)^{2 \lambda-3} ; \lambda \geq 2,
$$

and $p^{\text {near }}(\lambda<2)=1$. If the first patch contains $k_{1}$ ligands and the second patch $k_{2}$ ligands, the expected number of distinct configurations with lambda bonds also contains combinatorics of choosing $\lambda$ bonds out of the $k_{1}$ and $k_{2}$ ligands from the two patches

$$
\left\langle n^{\mathrm{cnf}}\right\rangle=\left(\begin{array}{c}
k_{1} \\
\lambda
\end{array}\right)\left(\begin{array}{c}
k_{2} \\
\lambda
\end{array}\right) \lambda ! \cdot p^{\text {near }}(\lambda) .
$$

The probability that a given interacting patch pair is expected to have at least one suitable bonding configuration is approximated as $p^{\text {near }}\left(k_{1}, k_{2}, \lambda\right) \approx \min \left[1,\left\langle n^{\text {cnf }}\right\rangle\right]$. 
Finally, the above equation is combined with the Poisson distribution of ligand numbers in patches discussed above to obtain the probability for the expected number of suitable patch pairs as a function of the number of formed bonds $\lambda$ :

$$
\left\langle n^{p p}\right\rangle_{\lambda}=\sum_{k_{1}} \sum_{k_{2}}\left\langle n^{p p}\right\rangle_{k_{1}, k_{2}} p^{\text {near }}\left(k_{1}, k_{2}, \lambda\right)
$$

This expression is a sum over all possible patch configurations (given by $k_{1}$ and $k_{2}$ ), $\left\langle n^{p p}\right\rangle_{k_{1}, k_{2}}$ is the expected number of such pairs of patches and $p^{\text {near }}\left(k_{1}, k_{2}, \lambda\right)$ specifies the probability that the given patch pair has a suitable lateral configuration of ligands. The limiting number of bonds $\lambda^{*}$ is determined by requiring that at least one such patch pair exists $\left\langle n^{p p}\right\rangle_{\lambda^{*}}=1$. This condition yields a self-consistent relation, which we solve to obtain the limiting number of bonds

$$
\lambda^{*}=\lambda^{*}\left(N, R^{*}\right)
$$

as a function of two dimensionless parameters: the total number of ligands on the particles $N$ and the particle radius $R^{*}=R_{p} / \delta x$ normalised by the typical bond extension $\delta x$. Note that for purely harmonic bonds $\delta x=(\beta k)^{-1 / 2}$, harmonic behaviour is expected when individual ligands are connected to the particle's surface via a flexible linker (e.g. a PEG-linker). The above derivation of $\lambda^{*}$, however, is also expected to be directly relevant, at least qualitatively, for rigid rod-like linkers usually employed in DNA-coated colloids [2].

The phase diagram on Figure 4a) shows this limiting value of the number of the bonds $\lambda^{*}$ as a function of the total number of ligands on the particles $N$. The shaded area below the curve outlines a region where the inter-colloidal interaction is expected to be homogenous.

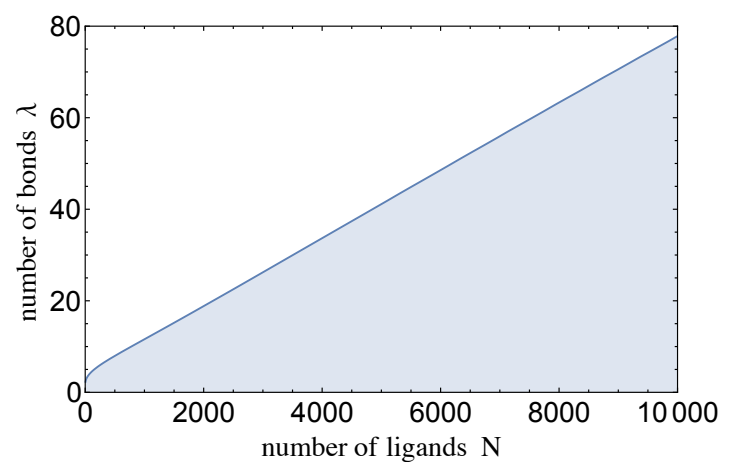

(a)

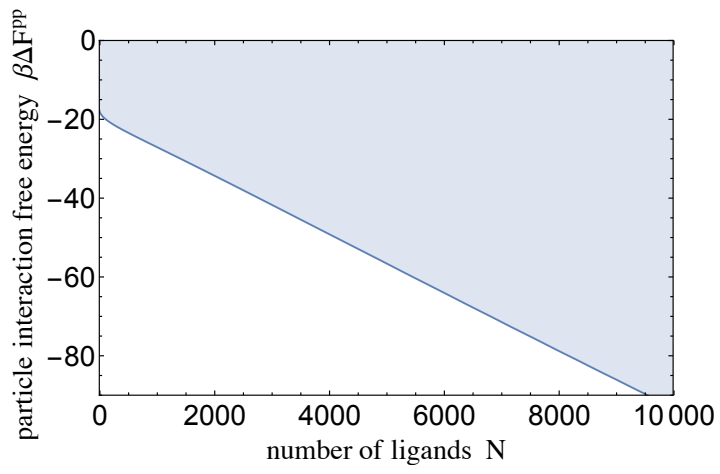

(b)

FIG. 4: Phase diagram showing the number of formed bonds (a) and the colloid interaction free energy

(b) for which the interaction is expected to be homogenous (shaded area) for Poisson distributed quenched ligands. The limiting number of bonds $\lambda^{*}$ and interaction free energy $\beta \Delta F^{p p *}$ are obtained using Eqs. (16) and (17), assuming a particle size of $R_{\mathrm{p}}=200 \mathrm{~nm}$ and bond length $\delta x=4 \mathrm{~nm}$. Such an effective bond length would would be expected if ligands are attached to a colloid via a PEG or dsDNA linker with size of about $\approx 2 \mathrm{~nm}$.

Experimentally, it is usually not straightforward to measure the number of formed bonds. Therefore, we also calculate the limiting particle-particle interaction free energy $\beta \Delta F^{p *}$ at which the average number of bonds equals the limiting number of bonds $\langle\lambda\rangle=\lambda^{*}$. Combining the equipartition relation, Eq. (8), with the mean-field expression, Eq. (10), we find that the limiting interaction is simply proportional to the limiting number of bonds

$$
\beta \Delta F^{p p *}=\lambda^{*}+\beta \Delta F_{0}^{p p} .
$$

We note that the reference is $\beta \Delta F_{0}^{p p}=\ln \left(4 \pi\left(2 R_{\mathrm{p}}\right)^{2} \delta x \rho_{0} N_{\mathrm{A}}\right)$. The result is shown on Figure $\left.4 \mathrm{~b}\right)$ for a particular particle size.

Furthermore, Figure 5 shows phase boundaries for various particle sizes as a function of the grafting density. For any point above the boundary the inter-particle interactions are expected to be homogenous. These theoretically determined phase boundaries match the simulation results shown in Figure 3. 


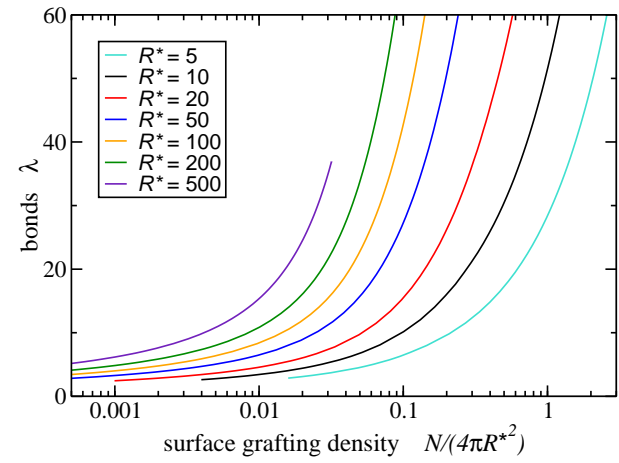

(a)

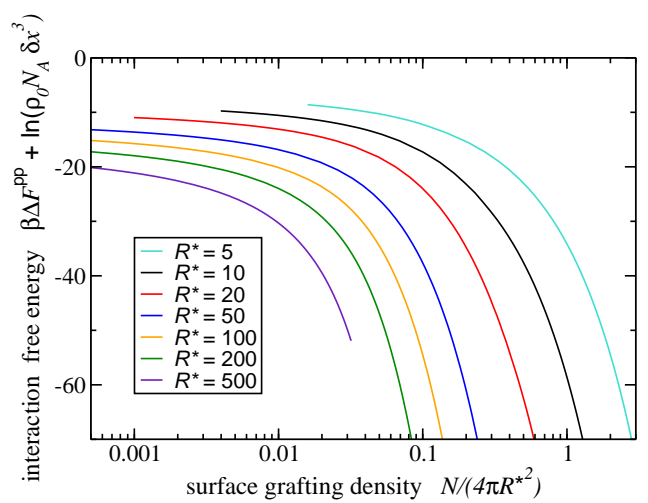

(b)

FIG. 5: Phase diagram showing (a) the limiting number of formed bonds $\lambda^{*}$, and (b) the limiting particle interaction free energy for which the particle pair interaction is expected to be homogenous.

Different curves correspond to different particle sizes $R^{*}=R_{\mathrm{p}} / \delta x$. For any point where $\lambda<\lambda^{*}$ or

equivalently, $\beta \Delta F^{p * *}>\beta \Delta F^{p p}$ the interaction is expected to be homogenous. The free energy reference is shifted in order for the phase diagram to be generally applicable for any bond size $\delta x$. The grafting density is expressed in the number of binders per $(\delta x)^{2}$. Phase boundaries are obtained using

Eqs. (14-17).

\section{DISCUSSION}

The typical length of the linkers used in experiments on ligand-coated colloids is a few nanometers and the reduced ligand grafting density is usually close to unity (one ligand per ligand size squared) [2]. From the phase diagram on Figure 5b) we can read that the interactions are sufficiently homogenous if the particles are larger than a few tens of nanometers and if the binding is not too strong and thus still reversible $\left(\Delta F^{p p} \gtrsim-30 k_{\mathrm{B}} T\right)$. In obtaining the phase diagram (Figure 5) we have assumed a Poisson distribution of the ligands, which is likely no longer justified if the grafting density is large $N /\left(4 \pi\left(R^{*}\right)^{2}\right) \geq 1$. In this case the analytical formulas, Eqs. (10) and (7), can only be applied if the number of formed bonds within the interacting patches is sufficiently low compared to the total number of ligands: $\lambda \ll N /\left(4 R^{*}\right)$.

On Figure 6 we provide a schematic phase diagram showing the region of validity of our analytical theory. The shaded area shows the region where the analytical expression (the "double exponential"), Eqs. (6-10), is expected to be valid. The $y$-axis shows the effective ligand excluded volume which influences the distribution of ligands on the particle's surface. All of our theoretical calculations were performed assuming ideal ligands where the number distribution is Poissonian. For Poisson distributed ligands the analytical theory is always valid. However, as demonstrated on Figure 3, even for uniformly distributed ligands (which would arise due to very strong excluded volume effects) the theory is valid if the number of formed bonds is low. We also show the phase boundaries where the pair-interaction in an ensemble of particles is expected to be homogenous. At uniform ligand distribution all particles are equal and the interaction is always homogenous. For Poisson distributed ligands, however, the limiting number of bonds is determined by plots on Figure 5 for quenched ligands, and for mobile ligands by requiring that the number if bonded ligands is a small fraction of the total. The boundary points determined in the Poisson and uniform limit are tentatively joined by straight lines to obtain a phase plot.

In the overlap of the two regions, the shaded region to the left of the dashed line, both the validity of double exponential and homogeneity of interactions is ensured and therefore the simple double exponential formula, Eq. (7), can be applied to describe the effective bonding interactions. For the parameter range outside of this region more involved procedures can be used to obtain the interaction free energy, e.g. Refs. [7-9] which rely on self consistently solving a set of coupled equations. 


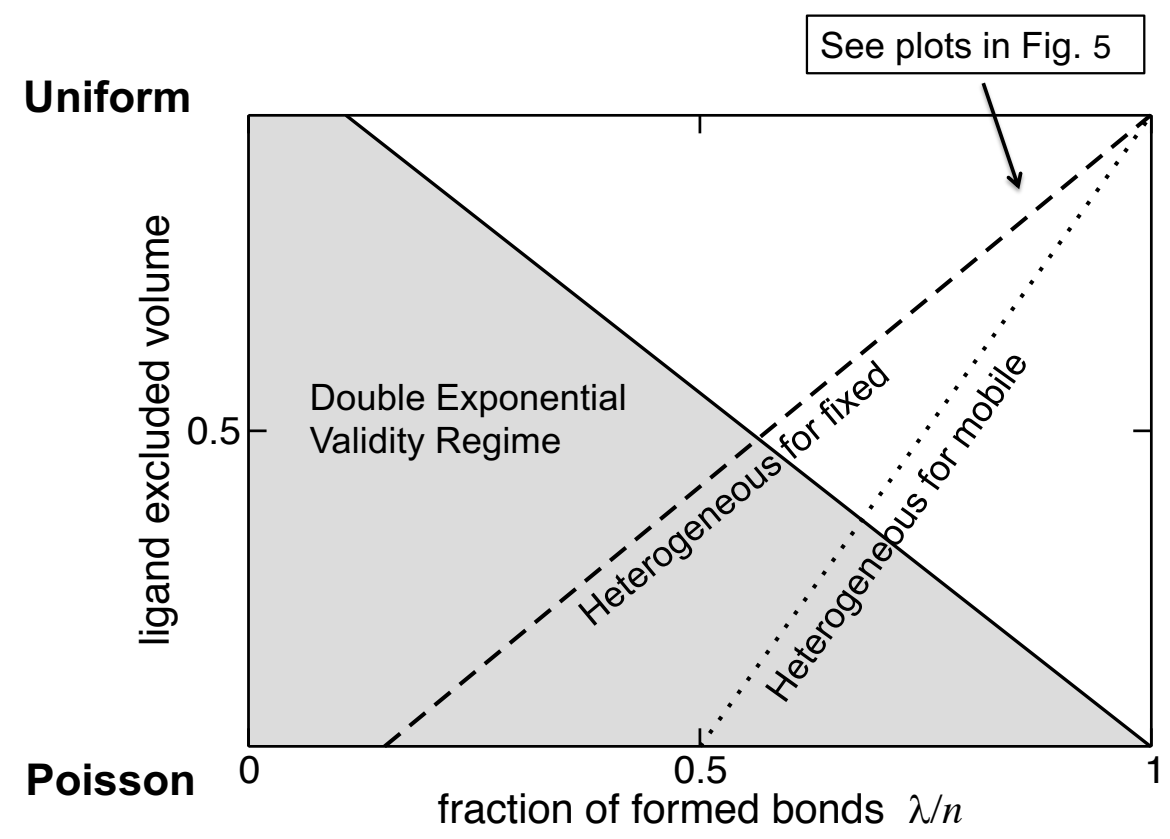

FIG. 6: Schematic phase diagram showing the regime of validity of the analytical expression for mean association strength between particles. The shaded region is where the analytical expression, Eq. (7), is justified (low binding strength and/or Poisson distributed ligands). The dashed and dotted lines mark the region boundary where the interactions are expected to be homogeneous according to the criterium defined in the text for two different grafting realisations: mobile (dotted line) and quenched ligands

(dashed line).

\section{CONCLUSIONS}

In conclusion, we considered particles coated with ideal binders, derived a simple analytical formula for the potential of mean force and for the pair interaction free energy due to bond formation. The analytical formula is generalised to multiple types of binders as well as multimeric complexes. We show that, quite generally, the pair interaction free energy in units of the thermal energy is equal to the expected number of formed bonds ("bridges") between the two particles. This result is analogous to equipartition theorem; each distinct bridge between the two particles provides one $k_{\mathrm{B}} T$ to the pair interaction free energy.

Furthermore, the effects of quenched disorder were analysed; how fixed, but randomly distributed, binder grafting points lead to heterogeneity of interactions. We also provided phase diagrams revealing the parameter range where the particle pair interaction is expected to be homogenous, as well as, mapped out a tentative phase diagrams clearly defining the parameter range where the analytical formula applies. Analytical results were verified and extended using Monte Carlo free energy calculations. We believe that the work presented shall provide a useful tool for designing experimental system involving bonding interactions.

\section{Acknowledgments}

We acknowledge fruitful discussion with Daan Frenkel, Stefano Angioletti-Uberti, Bortolo M. Mogneti and James D. Farrell, as well as the anonymous referee for useful suggestions. The work was supported by the Herchel Smith scholarship (T.C.), the CAS PIFI fellowship (T.C.) and by the European Union's 
Horizon 2020 programme under ETN grant 674979-NANOTRANS (J.D.).

[1] Chad A. Mirkin, Robert L. Letsinger, Robert C. Mucic, and James J. Storhoff. A dna-based method for rationally assembling nanoparticles into macroscopic materials. Nature, 382:607 EP -, 081996.

[2] Lorenzo Di Michele and Erika Eiser. Developments in understanding and controlling self assembly of dnafunctionalized colloids. Phys. Chem. Chem. Phys., 15:3115-3129, 2013.

[3] W. Benjamin Rogers and John C. Crocker. Direct measurements of dna-mediated colloidal interactions and their quantitative modeling. Proceedings of the National Academy of Sciences, 108(38):15687-15692, 2011.

[4] Stef A. J. van der Meulen and Mirjam E. Leunissen. Solid colloids with surface-mobile dna linkers. Journal of the American Chemical Society, 135(40):15129-15134, 2013. PMID: 24040916.

[5] Yu Wang, Yufeng Wang, Xiaolong Zheng, Étienne Ducrot, Jeremy S. Yodh, Marcus Weck, and David J. Pine. Crystallization of dna-coated colloids. Nature Communications, 6:7253 EP -, 062015.

[6] Stefano Angioletti-Uberti, Bortolo M. Mognetti, and Daan Frenkel. Theory and simulation of dna-coated colloids: a guide for rational design. Phys. Chem. Chem. Phys., 18:6373-6393, 2016.

[7] Patrick Varilly, Stefano Angioletti-Uberti, Bortolo M. Mognetti, and Daan Frenkel. A general theory of dna-mediated and other valence-limited colloidal interactions. The Journal of Chemical Physics, 137(9), 2012.

[8] Stefano Angioletti-Uberti, Patrick Varilly, Bortolo M. Mognetti, Alexei V. Tkachenko, and Daan Frenkel. Communication: A simple analytical formula for the free energy of ligand-receptor-mediated interactions. The Journal of Chemical Physics, 138(2), 2013.

[9] Stefano Angioletti-Uberti, Patrick Varilly, Bortolo M. Mognetti, and Daan Frenkel. Mobile linkers on dnacoated colloids: Valency without patches. Phys. Rev. Lett., 113:128303, Sep 2014.

[10] Nicholas B. Tito, Stefano Angioletti-Uberti, and Daan Frenkel. Communication: Simple approach for calculating the binding free energy of a multivalent particle. The Journal of Chemical Physics, 144(16), 2016.

[11] Lorenzo Di Michele, Stephan J. Bachmann, Lucia Parolini, and Bortolo M. Mognetti. Communication: Free energy of ligand-receptor systems forming multimeric complexes. The Journal of Chemical Physics, 144(16):161104, 2016.

[12] Omar A. Amjad, Bortolo M. Mognetti, Pietro Cicuta, and Lorenzo Di Michele. Membrane adhesion through bridging by multimeric ligands. Langmuir, 33(5):1139-1146, 2017. PMID: 28068766.

[13] David J. Wales and Sidika Ulker. Structure and dynamics of spherical crystals characterized for the thomson problem. Phys. Rev. B, 74:212101, Dec 2006.

[14] Tine Curk, Jure Dobnikar, and Daan Frenkel. Optimal multivalent targeting of membranes with many distinct receptors. Proc. Natl. Acad. Sci. U.S.A., pages 7210-7215, 2017.

[15] Pavel I. Kitov and David R. Bundle. On the nature of the multivalency effect: A thermodynamic model. Journal of the American Chemical Society, 125(52):16271-16284, 2003. PMID: 14692768.

[16] Francisco J Martinez-Veracoechea and Daan Frenkel. Designing super selectivity in multivalent nano-particle binding. Proceedings of the National Academy of Sciences of the United States of America, 108(27):1096310968, 2011.

[17] Paul L. Biancaniello, Anthony J. Kim, and John C. Crocker. Colloidal interactions and self-assembly using dna hybridization. Phys. Rev. Lett., 94:058302, Feb 2005.

[18] Ian C. Jenkins, John C. Crocker, and Talid Sinno. Interaction heterogeneity can favorably impact colloidal crystal nucleation. Phys. Rev. Lett., 119:178002, Oct 2017.

[19] D. Frenkel and B. Smit. Understanding Molecular Simulation: From Algorithms to Applications. Computational science series. Elsevier Science, 2001.

[20] Fugao Wang and D. P. Landau. Efficient, multiple-range random walk algorithm to calculate the density of states. Phys. Rev. Lett., 86:2050-2053, 2001. 